\title{
New Molecular Markers in Breast Cancer; Piwi-like protein-2 (PIWIL-2) and DEAD Box Helicase-4 (DDX-4) genes
}

\author{
(D) Murat ÇAĞ, 1 (D) Yeşim ÖZDEMIR, ${ }^{2}$ (1) Yusuf ÖZKUL³ \\ 'Department of Hepatopancreaticobilier and Transplantation, Strasbourg University, Strasbourg-Fransa \\ ${ }^{2}$ Department of Medical Genetic, Üsküdar University, İstanbul-Turkey \\ ${ }^{3}$ Department of Medical Genetic, Erciyes University, Kayseri-Turkey
}

\begin{abstract}
OBJECTIVE
Breast cancer, which basically is the unchecked proliferation of the epithelial cells covering the ductus and the lobules of the breast, is the most common form of cancer in women and ranks second after lung cancer among total deaths from that disease. As all other carcinomas, mamarian cancer results from the progressive accumulation of genetic anomalies. Several genes exist that have been associated positively with breast cancer. We aimed to research the molecular markers about breast cancer's etiology, screening, treatment, and its follow-up.
\end{abstract}

\section{METHODS}

Invasive ductal carcinoma from tumoral paraffin tissues and normal breast tissues taken from breast reduction surgeries was used in the study. The study examines the expressions of the PIWIL-2, PIWIL-1, DICER-1, and DDX-4 genes in the invasive ductal carcinoma of the breast.

\section{RESULTS}

As predicted, PIWIL-2 and DDX4 expressions were found to be significantly higher compared to normal tissues. PIWIL-1 expression, on the other hand, was recorded at borderline levels of significance. The expression of DICER-1, a gene responsible for the formation and maturation of microRNAs (miRNAs), revealed no significant difference.

\section{CONCLUSION}

It was concluded that the increased expression of PIWIL-2 and DDX-4 in breast cancer cases that are not detectable in normal tissue enables its use as a marker in the diagnosis and treatment of mamarian cancer and the post-therapy follow-up of the disease. It appears to be more informative to study the specific miRNAs of the gene rather than its expression to discern the function of the DICER-1 gene in breast cancer.

Keywords: Breast cancer; DDX-4; DICER-1; invasive ductal carcinoma; PIWIL-2; real-time polymerase chain reaction. Copyright $\odot$ 2021, Turkish Society for Radiation Oncology

\section{Introduction}

Breast cancer is the most frequent cancer among women and second leading cause of cancer death after lung cancer. The average lifetime risk of developing breast cancer is $12.4 \%$ or one in eight among women and the risk of death is $5 \%$. Breast cancer's mortality rate is estimated to increase in many parts of Europe by 2020.[1] Breast cancer, similar to other cancer types, originates from cancer stem cells. Proteins involved in 
self-renewal of stem cells play an essential role in tumor onset. This study aimed to investigate the role of PIWIL-2, a cancer stem cell gene, and the PIWIL-1, DICER-1, and DDX-4 genes that share certain pathways with this gene in the development of breast cancer.

At the cellular level, the development of cancer is viewed as a multistep process. At every step, important genetic processes are thought to occur that characterizes the cell as a cancer cell. As a result of these changes, alterations may occur in the expression of genes or in the function of gene products that cause tumor formation or progression.

Mutations or overproduction of receptors or proteins in signaling pathways cause cells to become independent from growth factors and other mitotic factors, thereby causing uncontrolled cell division. It is known that the histologically similar tumors have clinically different outcomes. Moreover, tumors with no established differences based on histological analysis can respond to treatments differently.[2]

Breast cancer is a heterogeneous disease, resulting from the progressive accumulation of genetic abnormalities, including point mutations, chromosomal instability, aneuploidies, deletions, rearrangements (chromothripsis), translocations, and duplications. These situations of DNA cause damage of DNA repair and telomere dysfunction.[3,4] All these conditions result in increased/decreased gene expression and epigenetic modifications. Classification of breast cancer according to the gene expression pattern was performed by Perou et al. and Sorlie et al.[5,6] Gene expression and DNA methylation profiling and other epigenetic modifications have been interpreted in breast cancer tissues with the aim to better refine the molecular classification and improve the prognostic and predictive abilities in the clinical areas. In addition, some studies subdivided the breast cancer patients with high probability of metastasis according to tumor gene expression profiles and planned to use this impersonalized treatment and clinical management of patients. [7-9]

Piwi genes have been preserved during evolution, and stem cells of different organisms ranging from the Arabidopsis plant to human are known to play a role in the regeneration, gametogenesis, and RNA interference. Piwi germline is involved as an autonomic organizer in stem cell division. PIWIL-2 is a member of the Argonaute protein family. This gene family has been revealed in studies to play a key role in gene silencing through small RNAs. The Piwi gene family contains two domains known as PAZ and Piwi, which are responsible for "stem-cell self-renewal, RNA silencing and transla- tional regulation." PIWIL-2, a member of the Piwi gene family, was demonstrated to be first expressed only in testicular tissue among normal tissues. In spermatogenesis, it plays a fundamental role in differentiation of spermatocytes, germ cells, and spermatogonia stem cells. It is thought to have a similar function during oogenesis in women. PIWIL-2 has a key role in regulation of proliferation and apoptosis in signal transduction pathways in cancer stem cells. Overexpression of this gene in germ cells leads to germ cell malignancies. This finding suggests that PIWIL-2 may cause the same effect in other stem cells including cancer development.

To understand the role of PIWIL-2 in different organs, northern blot analysis was performed in major adult organs and among the normal adult human tissues (spleen thymus, prostate, testis, ovary, small intestine, colon, peripheral blood, and leukocytes) analyzed, the expression of PIWIL-2 protein was observed only in the testis. In immunohistochemical studies, PIWIL-2 expression was detected in benign and malignant lesions of the breast. No difference in expression was noted between different breast cancer types. However, no expression was detected in benign lesions of the breast including fibroadenoma and fibrocystic disease. It is not clear why and how PIWIL-2 is activated in cancer stem cells while it is silent in somatic cells. Since this gene serves at a critical point at the beginning of DNA repair mechanisms, the primary reason for activation of the gene may be the initiation of DNA repair to stop oncogenesis. To confirm this hypothesis in invasive ductal carcinomas (IDC) of the breast, the molecular pathogenesis of IDCs will be elucidated by examining the expressions in the four genes as compared to the normal tissue and with each other.[10-13]

DICER is a key ribonuclease in microRNA (miRNA) and short interfering RNAs (siRNA) biosynthesis during mammalian development and cell differentiation. Recent studies have revealed that DICER-1 may also be involved in oncogenesis. Death occurred as a result of depletion of stem cells in DICER gene-silenced mice. Embryonic stem cells with DICER deficiency can survive but lose their differentiation and proliferation ability. Therefore, DICER-1 is the basis for stem cells' regeneration and proliferation. DICER is involved in the metabolic processes of Piwi-interacting RNAs (piRNAs), which are 24-30 nucleotide RNA fragments that are formed by an independent mechanism and are primarily composed of transposon and other repeating sequence elements. Besides suppressing transposons, piRNAs are also involved in some functional positions and translational regulation, possibly during meiosis. It 
also plays a role in the stabilization of miRNA and the formation of the chromatid body. Small RNAs such as siRNAs, miRNAs, or piRNAs bind to specific binding sites, leading to silencing and destruction in the target mRNA. Small RNAs and Argonaute proteins have been found in high eukaryotes and have very different functions such as transposon silencing, cell differentiation, and embryonic development.[14] Argonaute protein has been preserved during the evolution process and phylogenetically divided into the Ago and Piwi subclasses. The expression of the Piwi protein is limited to germline cell lines and is associated with piRNAs, which enables the silencing of transposons. Although Argonaute protein functions are understood from various aspects, there are many Argonaute proteins that have not yet been identified. This study was carried out in accordance with the approval of "Erciyes University Clinical Research Ethics Committee" with date February 10, 2012 and no: 2012/130.

\section{Materials and Methods}

A total of 41 tumor tissues diagnosed as IDC and 14 normal breast tissues (NBT) were included in the study. All participants written and signed consents were taken accordingly ethical committees guidelines by the researchers. The tissue samples of the study and control groups were obtained from the Erciyes University Faculty of Medicine Pathology Department Archive. All tissues were evaluated for age and estrogen receptor (ER), progesterone receptor (PR), lymph node metastasis, ki67 , and c-erbB-2 positivity. The patients were grouped according to cell proliferation index ki-67, poor prognostic marker c-erbB-2, and lymph node metastasis.

All participants were females aged $35-60$ years. The study group was selected from patients operated for breast cancer and the control group from those who underwent mammoplasty (breast reduction). Patients diagnosed as "Invasive Ductal Carcinoma" in the pathology report were included in the study group and those identified as "Normal breast tissue" in the pathology report served as the control group. Males and patients with any pathological diagnosis other than or in addition to "Invasive Ductal Carcinoma and Normal Breast Tissue" in fine-needle aspiration biopsy materials were excluded from the study.

In this study, the expressions of PIWIL-2, PIWIL-1, DICER-1, and DDX-4 genes in the IDC of the breast were analyzed by real-time polymerase chain reaction (RT-PCR) method. For the expression analysis, betaactin (ACTB), a housekeeping gene was used as the control gene, and NBT samples obtained from mammoplasty operations were used as the control tissue sample. RNeasy FFPE Kit was used for RNA isolation from the tissues. The c-DNA synthesis and pre-amplification of the RNAs were performed with RT2 PreAMP c-DNA Synthesis Kit. RT-PCR method was applied after pre-amplification.

\section{Results}

Of the 55 cases included in the study, 41 had a pathology report of "invasive ductal carcinoma" and 14 cases had a report of "normal breast tissue" after mammoplasty. When tissues diagnosed with IDC were evaluated according to grade, $51 \%$ were Grade 3 and $49 \%$ were Grade 2. Lymph node excision was noted in 27 (65.9\%) of 41 cases with IDC. While $73 \%$ of these cases had at least one metastatic lymph node, $34.1 \%$ had no metastatic lymph nodes.

In tissues diagnosed with IDC, the ER positivity rate was $70 \%$ and $\mathrm{PR}$ positivity rate was $58 \%$. The c-erbB-2 was positive in $46 \%$ of tumor tissues and negative in $53 \%$. The ki- 67 positivity was very high including $95 \%$ of the cases. Figures $1 \mathrm{a}, \mathrm{b}, 2 \mathrm{a}, \mathrm{b}$, and 3 show the expression curves of the PIWIL1-2, DICER1, and DDX4 genes. Our findings revealed significant interindividual differences in the expression levels of PIWIL-1, PIWIL-2, DICER-1, and DDX-4 genes in IDC. Based on this finding, it can be considered that each cancer tissue exhibits very different functional and genetic features. In addition, there are many factors that we could not evaluated with potential effects on the physiological status of the patient, the cancer tissues of independent patients, and the expressions. Due to these differences, the correlation of expression levels with lymph node metastasis, ki-67, ER and PR, and cerbB-2 positivity could not be analyzed statistically.

According to the Delta-Delta Ct method, that we used to compare the expression levels in normal tissues and the tissues with IDC, the level of expression in all included genes of the study had increased in IDC tissues compared to NBT, as presented in Table 1. On the basis of $\mathrm{P}$ values obtained in statistical analysis with the Mann-Whitney U-test, it can be stated that the increase in DDX-4 and PIWIL-2 was statistically significant $(\mathrm{p}<0.05)$, while a near-significant trend was noted for the increase in the expression of the PIWIL-1 gene implicating the likelihood of achieving significance on analysis of an increased number of tissue samples and the increase in the DICER-1 gene was not significant statistically (Table 2). 


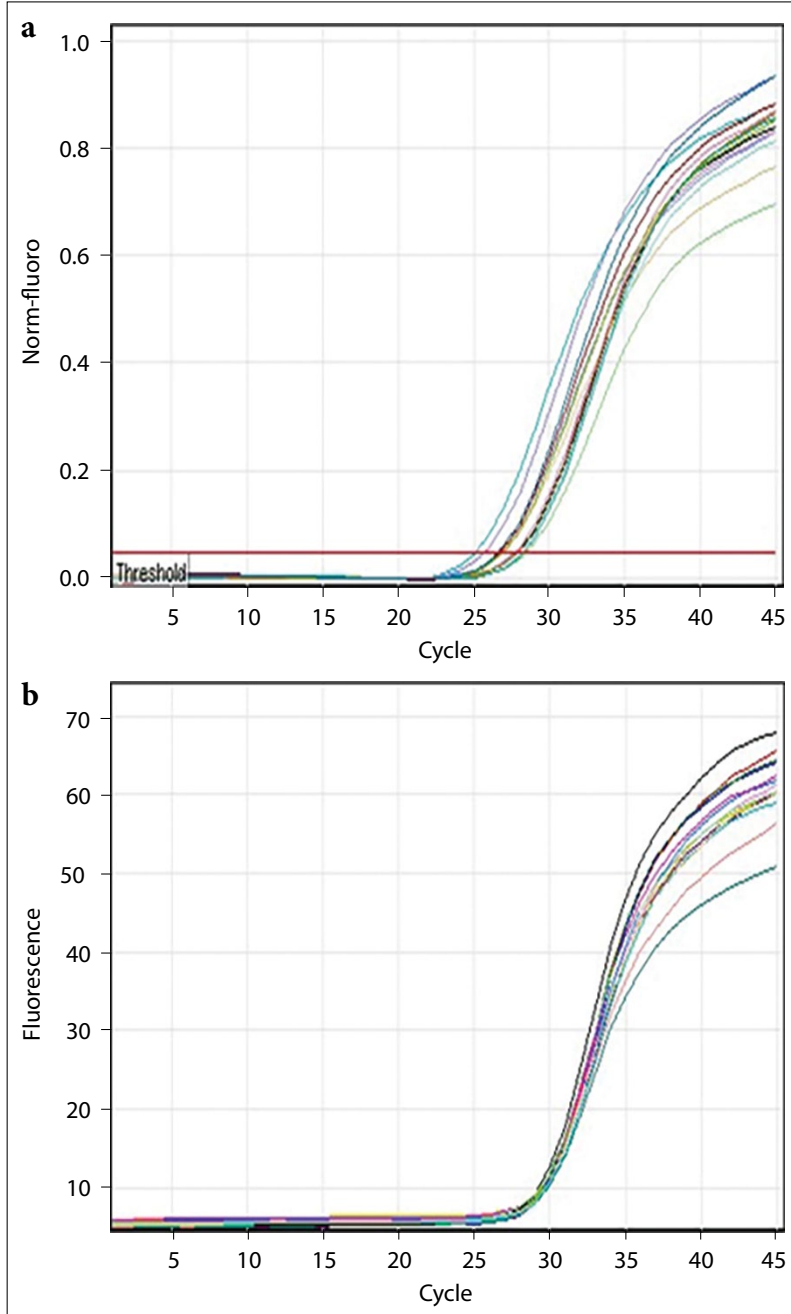

Fig. 1. (a, b) The expression curves of ACTB and PIWIL-2 genes.

\section{Discussion}

In a meta-analysis made by Mentis et al., PIWI family expression was a prognostic marker in cancer: Higher piwil 1 and lower piwil 4 expression levels show potentially indicate worse prognosis in cancer.[15] Li et al.[16] found that PIWIL-2 expression negatively affected the 5 -year survival and increased the rate of metastasis in colorectal cancers. In the study of Greither et al. concerning the Piwi proteins in patients with soft-tissue tumors, authors reported a significant increase in PIWIL-2 and PIWIL-4 mRNA levels only in female patients with no significant change in male patients, along with the association of the increase in PIWIL-2 and PIWIL-3 with poor survival.[17] Wang et al.[18] reported significantly increased PIWIL1-4 expressions in tumor tissues compared to surrounding tissues in human gastric cancer.
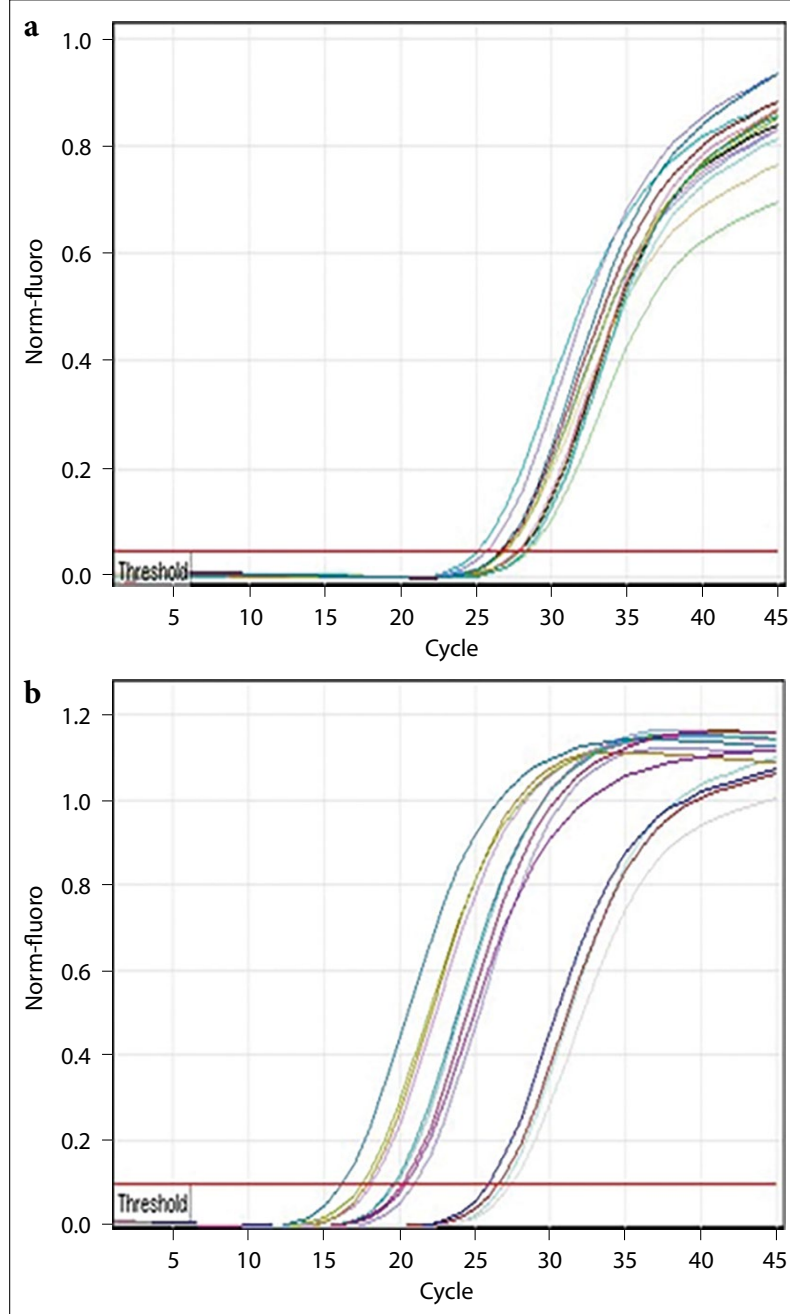

Fig. 2. (a, b) The expression curves of PIWIL-1 and DICER-1 genes.

They also indicated poorer prognosis in patients with high versus low PIWIL-1 and PIWIL-2 expressions. Tosun et al.[19] demonstrated in their study on prostate cancer papillary that PIWIL-2 gene expression in blood was observed to be significantly higher among patient with International Society of Urological Pathology (ISUP) of $>3$ than among those with ISUP of $\leq 3 \mathrm{~s}$.

In a study by Nickpour et al. on bladder cancers, PIWIL-2 expression was reported to be at least 240 times lower in all cell series when compared to levels in the testicular tissue. Such a low level of PIWIL-2 expression in most tissues may enable this gene to be considered as a tumor marker in the future. However, the same researchers showed that PIWIL-2 had no role in carcinogenesis in bladder cancer[20] cytoplasmic but not nuclear PIWIL2 expression was reported to be a predictor of mortality in bladder cancer.[21] 


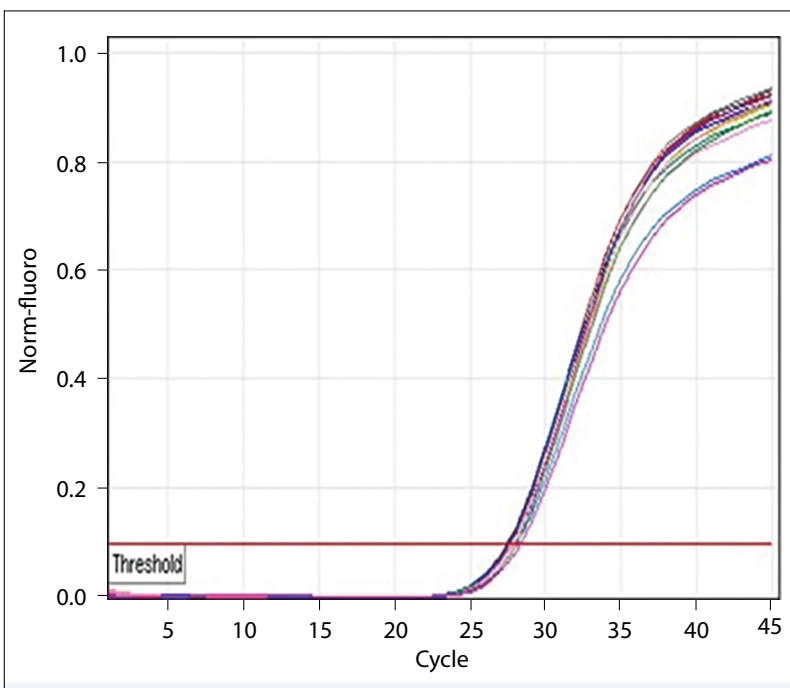

Fig. 3. The expression curve of the DDX-4 gene.

Therefore, the authors suggested that Piwil 2 may be a novel biomarker for breast cancer.[22]

Lee et al. found that PIWIL-2 inhibits apoptosis by activating the STAT3-BCL-XL pathway that has expression in tumors.[10] In our study, PIWIL-2 gene expression was found to be significantly higher in the IDC of the breast than normal tissues as expected. Expression of PIWIL-1 showed a near-significant trend, possibly due to low number of samples in statistical terms. Since PIWIL-2 is a gene that has no expression in normal tissues other than the testicular tissue, any existence of PIWIL-2 expression may suggest the presence of a cancer cell in that tissue or any part of the body. This may allow PIWIL-2 to be used as a "tumor marker" in the future. We could not find any cancer studies related to DDX-4 gene in the literature. In one study conducted in 2002, it has been considered to be a marker that can determine malignancy in reproductive organ tumors.[23] This gene is more effective in the development and function of germ cells. As the DDX-4 is not expressed in normal tissues, and expressed only in the testicles, and has similarities with PIWIL-2, it may suggest that the DDX-4 gene that we included in our study was working together at the differentiation stage by affecting PIWIL-2 positively. On the other hand, the absence of expression in normal tissue and existence of similarly increased expressions of both genes in cancer tissue support our hypothesis. This gene, like PIWIL-2, may be a marker for cell proliferation and oncogenesis.

DICER-1 is a gene involved in the formation and maturation of miRNAs. At the beginning of our

\begin{tabular}{lccc} 
Table 1 & \multirow{2}{c}{$\begin{array}{c}\text { Delta-delta Ct values and standard deviation of } \\
\text { the genes investigated }\end{array}$} \\
\cline { 3 - 4 } Position & Gene & \multicolumn{2}{c}{$\mathbf{2 \wedge}$ (-Avg. [Delta (Ct)]) } \\
\cline { 3 - 4 } & & IDC & Control \\
group & group \\
\hline A01 & DDX4 & 0.015678 & 0.003514 \\
A02 & DICER1 & 0.560171 & 0.419409 \\
A03 & PIWIL1 & 0.119631 & 0.094873 \\
A04 & PIWIL2 & 0.027532 & 0.010693 \\
A05 & ACTB & 1 & 1 \\
\hline
\end{tabular}

Ct: Threshold cycle; IDC: Internal data control; DDX4: DEAD Box Helicase-4; DICER1: Double-stranded RNA-specific endoribonuclease; PIWIL1-2: Piwi-like protein-1-2; ACTB: Actin beta

Table 2 Statistical findings between the genes according to Mann-Whitney U-test, p-values

\begin{tabular}{lcc} 
Position & Genes & $\begin{array}{c}\text { p (compared } \\
\text { to control group) }\end{array}$ \\
\hline $\mathrm{A} 01$ & DDX4 & 0.027 \\
$\mathrm{~A} 02$ & $\mathrm{DICER1}$ & 0.657 \\
$\mathrm{~A} 03$ & $\mathrm{PIWIL1}$ & 0.073 \\
$\mathrm{~A} 04$ & PIWIL2 & 0.039 \\
$\mathrm{~A} 05$ & $\mathrm{ACTB}$ & 0 \\
\hline
\end{tabular}

DDX4: Piwi-like protein-2; DICER1: Double-stranded RNA-specific endoribonuclease; PIWIL1-2: Piwi-like protein-1-2; ACTB: Actin beta

study, we expected the expression of this gene to be increased in IDC compared to NBT, because the miRNAs' crucial role in cancer pathogenesis could not be denied. In our study, we did not find a statistical difference in the expression of the DICER-1 gene in IDC group compared to normal individuals. As our research progressed further, we considered this as a normal finding, since DICER-1 contributes not only to carcinogenesis but also to the formation of miRNAs in hundreds of different mechanisms. Therefore, the presence of specific miRNAs formed by this gene and their relation with the breast cancer should be planned to be investigated by another study, rather than global expression of this gene in the cell. The identification of the genes that are involved in tumorigenesis is possible by genomic and transcriptomic comparisons of different tumor samples with normal tissue samples and with each other and by the use of genomic information in oncology. Data of these studies will enable to categorize the tumors as subgroups and to use them as personalized treatments. 


\section{Conclusion}

In this study, the interplay between PIWIL-1, PIWIL-2, DICER-1, and DDX-4 genes in breast cancer was addressed. In summary, our findings indicate;

1. Among these genes, the expression of PIWIL-2 and DDX-4 was not evident or very low in NBT, while their expression increased in breast cancer tissue. PIWIL-2 and DDX-4 genes work in parallel. Although one of them was increased with the increase in other, the increase in DICER-1 was not found to be significant among the groups. Given that both PIWIL-2 and DDX-4 genes are increasingly expressed in breast cancer despite not expressed in normal tissue, it seems likely that they can be used as markers in the diagnosis and treatment of breast cancer and follow-up of disease after treatment

2. Considering no change in the expression of DICER-1 between normal and IDC tissues, we think that the miRNAs that are involved in the production of the gene, other than the gene expression, should be investigated

3. Until the genomic revolution, the tumors were classified according to two criteria; localization (the region of the tumor originated) and the appearance of the tumor (histological structure). These two criteria are still in use today for the determination of the prognosis and application of the best proper treatment.

Nevertheless, it has been known for long that histologically similar tumors have different clinical outcomes. In addition to this information, the new tumor taxonomy, in which the presence of genetic lesions is the major criteria, will enable the likelihood of better diagnosis and individualized treatment plan by means of the genome-based information. The gene expressions and proteins will be included in the new classification as a discriminating criterion besides to the other two criteria due to contribution of the analysis of gene expression profiles in the diagnosis and treatment.

These results will help to highlight the molecular pathogenesis of breast cancer, while possibly one or more of these genes can be used as molecular markers that can be determined before the oncogenesis in the diagnosis of IDC.

Peer-review: Externally peer-reviewed.

Conflict of Interest: The authors declare that they have no conflict of interest.
Ethics Committee Approval: The study was approved by the Erciyes University Clinical Research Ethics Committee (No: 2012/130, Date:10/02/2012).

Financial Support: The authors declared that this study has received no financial support.

Authorship contributions: Concept - Y. Özkul; Design Y.Ö.; Supervision - Y.Ö.; Funding - Y.Ö.; Materials - M.Ç., Y. Özkul; Data collection and/or processing - Y.Ö.; Data analysis and/or interpretation - M.Ç., Y. Özkul; Literature search - M.Ç.; Writing - M.Ç., Y. Özkul; Critical review - M.Ç.

\section{References}

1. Momenimovahed Z, Salehiniya H. Epidemiological characteristics of and risk factors for breast cancer in the world. Breast Cancer (Dove Med Press) 2019;11:151-64.

2. Bertucci F, Ng CK, Patsouris A, Droin N, Piscuoglio S, Carbuccia N, et al. Genomic characterization of metastatic breast cancers. Nature 2019;572(7767):E7.

3. Duijf $P H$, Nanayakkara D, Nones $K$, Srihari S, Kalimutho M, Khanna KK. Mechanisms of genomic instability in breast cancer. Trends Mol Med 2019;25(7):595-611.

4. Huang Y, Nayak S, Jankowitz R, Davidson NE, Oesterreich S. Epigenetics in breast cancer: what's new? Breast Cancer Res 2011;13(6):225.

5. Perou CM, Sorlie T, Eisen MB, van de Rijn M, Jeffrey SS, Rees CA, et al. Molecular portraits of human breast tumours. Nature 2000;406(6797):747-52.

6. Sorlie T, Perou CM, Tibshirani R, Aas T, Geisler $\mathrm{S}$, Johnsen $\mathrm{H}$, et al. Gene expression patterns of breast carcinomas distinguish tumor subclasses with clinical implications. Proc Natl Acad Sci USA 2001;98(19):10869-74.

7. Pasculli B, Barbano R, Parrella P. Epigenetics of breast cancer: biology and clinical implication in the era of precision medicine. Semin Cancer Biol 2018;51:22-35.

8. Stefansson OA, Esteller M. Epigenetic modifications in breast cancer and their role in personalized medicine. Am J Pathol 2013;183(4):1052-63.

9. Paik S, Shak S, Tang G, Kim C, Baker J, Cronin M, et al. A multigene assay to predict recurrence of tamoxifentreated, node-negative breast cancer. N Engl J Med 2004;351(27):2817-26.

10. Hock J, Meister G. The Argonaute protein family. Genome Biol 2008;9(2):210.

11. Liu JJ, Shen R, Chen L, Ye Y, He G, Hua K, et al. PIWIL2 is expressed in various stages of breast cancers and has the potential to be used as a novel biomarker. Int J Clin Exp Pathol 2010;3(4):328-37.

12. Ye Y, Yin DT, Chen L, Zhou Q, Shen R, He G, et al. 
Identification of PIWIL-2-Like (PL2L) proteins that promote tumorigenesis. PLoS One 2010;5(10):e13406.

13. Lee JH, Schutte D, Wulf G, Fuzesi L, Radzun HJ, Schweyer S, et al. Stem-cell protein PIWIL-2 is widely expressed in tumors and inhibits apoptosis through activation of Stat3/Bcl-XL pathway. Hum Mol Genet 2006;15(2):201-11.

14. Iwasaki YW, Siomi MC, Siomi H. PIWI-Interacting RNA: Its biogenesis and functions. Annu Rev Biochem 2015;84:405-33.

15. Mentis AF, Dardiotis E, Romas NA, Papavassiliou AG. PIWI family proteins as prognostic markers in cancer: a systematic review and meta-analysis. Cell Mol Life Sci 2020;77(12):2289-314.

16. Li D, Sun X, Yan D, Huang J, Luo Q, Tang H, et al. PIWIL-2 modulates the proliferation and metastasis of colon cancer via regulation of Matrix Metallopeptidase 9 transcriptional activity. Exp Biol Med (Maywood) 2012;237(10):1231-40.

17. Greither T, Koser F, Kappler M, Bache M, Lautenschlager C, Gobel S, et al. Expression of human Piwilike genes is associated with prognosis for soft tissue sarcoma patients. BMC Cancer 2012;12:272.
18. Wang Y, Liu Y, Shen X, Zhang X, Chen X, Yang C, et al. The PIWI protein acts as a predictive marker for human gastric cancer. Int J Clin Exp Pathol 2012;5(4):315-25.

19. Tosun H, Demirtaş A, Sönmez G, Tombul ST, Akalın $\mathrm{H}$, Özkul Y. Can the expression level of PIWIL 2 gene be a serum marker for prostate cancer? a single-center prospective study. Turk J Urol 2019;45(1):S22-5.

20. Nikpour P, Forouzandeh-Moghaddam M, Ziaee SA, Dokun OY, Schulz WA, Mowla SJ. absence of PIWIL2 (HILI) expression in human bladder cancer cell lines and tissues. Cancer Epidemiol 2009;33(3-4):271-5.

21. Taubert H, Wach S, Jung R, Pugia M, Keck B, Bertz $\mathrm{S}$, et al. Piwil 2 expression is correlated with diseasespecific and progression-free survival of chemotherapy-treated bladder cancer patients. Mol Med 2015;21(1):371-80.

22. Gainetdinov IV, Skvortsova YV, Stukacheva EA, Bychenko OS, Kondratieva SA, Zinovieva MV, et al. Expression profiles of PIWIL2 short isoforms differ in testicular germ cell tumors of various differentiation subtypes. PLoS One 2014;9(11):e112528.

23. Schudrowitz N, Takagi S, Wessel GM, Yajima M. Germline factor DDX4 functions in blood-derived cancer cell phenotypes. Cancer Sci 2017;108(8):1612-9. 